\title{
Leading organisational change: how we implemented a new electronic solution in a district general hospital
}

\author{
Authors: Sean Noronha* and Victoria Alner
}

\section{Background}

The background to this project was a growing district general hospital reliant on an increasingly outdated system of paper notes, drug charts and separate departments with idiosyncratic requesting procedures. Processes were cumbersome, non-auditable and open to systemic error.

The eCare solution would amalgamate these systems into single, auditable patient records, accessible from any trust computer, solving any issues with legibility or accountability. Our challenge was to train an entire hospital of healthcare professionals on eCare prior to 'go live', and transcribe all inpatient drug charts on the transition weekend.

\section{Methods}

As chief registrar, I was tasked with two specific outcomes:

> For $90 \%$ of junior doctors to have completed training prior to go-live: this was a collaborative effort between doctors, medical staffing and the transformation teams to ensure doctors could attend training without compromising patient care on the wards. Bespoke training sessions were used to deliver staff-specific needs, while grand rounds and audit half days were used to deliver the strategic vision behind the project.

> For $100 \%$ of drug charts to be transcribed during the cutover weekend: junior doctors from our existing staff pool were recruited for drug chart transcription at highly competitive locum rates. A bonus was offered to the doctor who transcribed the most drug charts.

\section{Results}

We achieved results of $93 \%$ of junior doctors completing training prior to the go live date and $100 \%$ of drug charts being transcribed. The success of the project could be attributed to junior doctor buyin, a competitive locum rate for transcription slots and the presence of a chief registrar with dedicated time to drive the project forwards.

\section{Conclusions}

The implementation of eCare was a transformational change affecting every healthcare professional in the trust, and we worked

Authors: Milton Keynes University Hospital NHS Foundation Trust *Royal College of Physicians chief registrar tirelessly with both internal and external stakeholders to ensure the smoothest transition possible. The junior doctors were identified as key to the success of the project, and great efforts were made to win over their hearts and minds, focusing on immediate gains such as never rewriting drug charts, ability to prescribe remotely, and not having to look for or decipher notes. With the clinical workforce on board, there is now cautious optimism as the project moves forwards into the next phase.

\section{Conflict of interest statement}

None declared. 